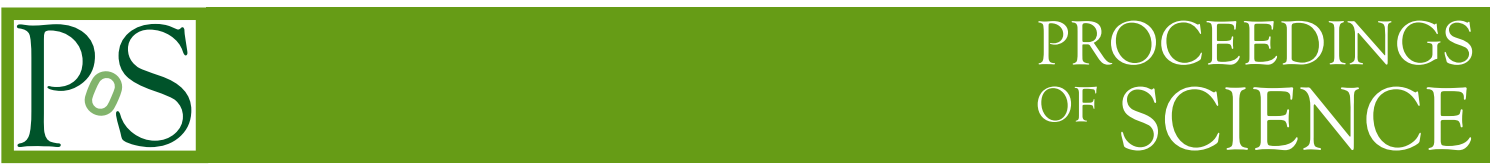

\title{
Hypernuclear decay pion spectroscopy at MAMI-C
}

\author{
Sho Nagao*† \\ Tohoku University \\ E-mail: nagaodlambda.phys.tohoku.ac.jp
}

\begin{abstract}
Measurements of absolute binding energies of hypernuclei contribute to a comprehensive understanding of the interaction between nucleons and hyperons ( $Y N$ interaction). Hypernuclear decay-pion spectroscopy is devised as a new method to deduce the absolute binding energy of light hypernuclei with an accuracy of $\sim 30 \mathrm{keV}$. The first feasibility experiment was performed in 2011 and the second experiment was performed in 2012 using the high intensity electron beam of the Mainz Microtron C (MAMI-C). We successfully identified the pionic decay events from ${ }_{\Lambda}^{4} \mathrm{H}$ using a positron absorber in the second experiment. However, as continuous pion background events from quasifree hyperons cannot be negligible, we learned that a collimator should be installed around the beam target to suppress the background events. We expect to suppress $70 \%$ background using the collimator. The next experiment is planed in early summer of 2014.
\end{abstract}

XV International Conference on Hadron Spectroscopy-Hadron 2013

4-8 November 2013

Nara, Japan

\footnotetext{
* Speaker.

$\dagger$ On behalf of the A1 hypernuclear collaboration: Patrick Achenbach, Naoki Arai, Carlos Ayerbe Gayoso, Ralph Böhm, Olga Borodina, Damir Bosnar, Vakkas Bozkurt, Luka Debenjak, Michael O. Distler, Anselm Esser, Ivica Friščič, Yuu Fujii, Toshiyuki Gogami, Mar Gómez Rodríguez, Satoshi Hirose, Hiroki Kanda, Masashi Kaneta, Eunhee Kim, Junichiro Kusaka, Amur Margaryan, Harald Merkel, Ulrich Müller, Satoshi N. Nakamura, Josef Pochodzalla, Christophe Rappold, Joerg Reinhold, Takehiko R. Saito, Alicia Sanchez Lorente, Salvador Sánchez Majos, Björn Sören Schlimme, Matthias Schoth, Florian Schulz, Concettina Sfienti, Simon Širca, Liguang Tang, Michaela Thiel, Kyo Tsukada, Daisuke Uchiyama
} 


\section{Introduction}

Investigations of nuclear force and hyperon force are essential for understandings of nuclear structure and high density matter such as a neutron star. Strengths of nuclear forces have been tuned very well to reproduce the rich $N N$ scattering data. These scattering data led to realistic $N N$ forces such as CD Bonn[1], AV18[2], Nijmegen I and II[3]. By contrast, strengths of hyperon forces are not yet well understood due to limited $Y N$ scattering data. Experimental data for energy structures of hypernuclei are important inputs to understand the hyperon force.

Precise measurements of binding energies in light hypernuclei have been performed by emulsion technique half a century ago [4]. This is precious data to constrain the $Y N$ interaction. A large charge symmetry breaking (CSB) effect of the $\Lambda N$ interaction was advocated with the results of emulsion data; nevertheless the effect is still under discussion. The starting point of this argument is a $350 \mathrm{keV}$ binding energy differences of ground states in $A=4$ iso-doublet hypernuclei namely, ${ }_{\Lambda}^{4} \mathrm{H}\left(0^{+}\right)$and ${ }_{\Lambda}^{4} \mathrm{He}\left(0^{+}\right)$. This energy difference is several times larger than the difference of the $A=3$ iso-doublet nuclei, ${ }^{3} \mathrm{H}$ and ${ }^{3} \mathrm{He}$, after a correction of the Coulomb effects [5]. Theoretical calculations with a mixing effect of $\Sigma$ hyperons reproduce the differences in the $A=4$ hypernuclear system; however, it is difficult to explain quantitatively [6] [7] and phenomenological $\Lambda N$ CSB potential was introduced to reproduce it [8].

Recently, the absolute binding energy of ${ }_{\Lambda}^{7} \mathrm{He}$ was provided by JLab E01-011 experiment using the $\left(\mathrm{e}, \mathrm{e}^{\prime} \mathrm{K}^{+}\right.$) reaction [9], so that the binding energies became complete in the $A=7$ iso-triplet hypernuclei ${ }_{\Lambda}^{7} \mathrm{He}\left(1 / 2^{+}\right),{ }_{\Lambda}^{7} \mathrm{Li}^{*}\left(1 / 2^{+}\right)$, and ${ }_{\Lambda}^{7} \mathrm{Be}\left(1 / 2^{+}\right)$[4] [10]. In addition, recent progress of cluster calculations made quantitative predictions of binding energies in the $A=7$ hypernuclei with CSB and without CSB effect which is necessary for reproducing the $A=4$ hypernuclear system [11]. According to the discussion of these works experimental data of $A=7$ hypernuclei may prefer the calculation without CSB though CSB is necessary to explain the mass difference of $A=4$ systems. The inconsistency indicated that phenomenological CSB potential might be too naive and motivated to measure again the binding energies of $A=4$ hypernuclear iso-doublet with the state-of-the-art experimental technique since they are the starting point of the $\Lambda N$ CSB argument.

\section{Overview of the hypernuclear decay pion spectroscopy}

The hypernuclear decay pion spectroscopy was designed to measure the binding energy of hypernuclear ground state with an accuracy of $30 \mathrm{keV}$ [12]. The mass of hypernuclei is deduced by detecting monochromatic pions from two-body decays of hypernuclei stopped in the target as follows,

$$
M_{\text {hyp }}=\sqrt{M_{\text {nucl }}^{2}+p_{\pi^{-}}^{2}}+\sqrt{M_{\pi^{-}}^{2}+p_{\pi^{-}}^{2}},
$$

where $M_{\text {hyp }}$ is mass of hypernucleus, $M_{\text {nucl }}$ mass of daughter nucleus, $M_{\pi^{-}}$mass of pion, and $p_{\pi^{-}}$ momentum of decay pion. As the uncertainties of the masses in the daughter nucleus and the pion are negligible comparing with the uncertainty of momentum, the precision of pion momentum measurement mainly determines the resolution of hypernuclei masses. A thin target and a high intensity beam are required to be minimized the energy straggling effect for decay pions with keeping high hypernuclei yield in the experiment. At least one high-resolution spectrometer is also 


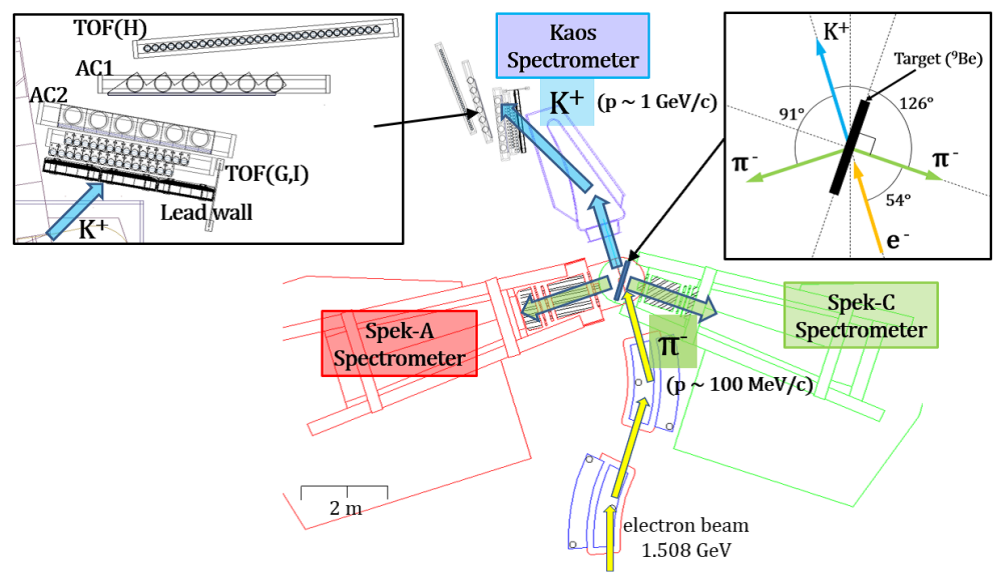

Figure 1: The experimental setup of hypernuclear decay pion spectroscopy in 2012. We irradiated 1.508 $\mathrm{GeV}$ electron beam to a ${ }^{9} \mathrm{Be}$ target. The target thickness was $120 \mu \mathrm{m}$, and it was tilted by $54^{\circ}$ with respect to the incident electron beam. Pions from hypernuclear decay were measured its momentum in Spek-A and Spek-C. The angle of Spek-C was adjusted by normal angle for the target to minimize the energy straggling effect of pion. Kaons were tagged in Kaos to identify the hyperon formation. Lead wall and second layer of aerogel Cherenkov detector (AC2) were newly installed to improve kaon identification in 2012 beam time.

required to measure the $\pi^{-}$momentum accurately. In addition, $K^{+}$is detected simultaneously to tag strangeness formation.

The MAMI-C accelerator can provide a continuous electron beam with a high intensity up to $\sim 100 \mu \mathrm{A}$. Furthermore, two well-studied high resolution spectrometers are installed in the A1 experimental hall: Spek-A and Spek-C [13], which have relative momentum resolution of $10^{-4}$ and $28 \mathrm{msr}$ large solid angle acceptance, simultaneously. The spectrometer for $\mathrm{K}^{+}$tagger, Kaos, is also installed, which has short central trajectories of $\sim 7 \mathrm{~m}$ and a large momentum acceptance of $\pm 25 \%$. For this reason, MAMI is suitable place to perform the hypernculear decay pion spectroscopy. We have performed the first experiment in 2011 and an updated experiment in 2012.

Figure 1 shows the experimental setup of hypernuclear decay pion spectroscopy in 2012. A beam target adopt $120 \mu \mathrm{m}$ thick ${ }^{9} \mathrm{Be}$, which was tilted by 54 degree with respect to the incident electron beam to get better hypernuclei yield and minimize the energy straggling effect of pion. Kaos spectrometer was installed at a very forward angle to keep a high yield of kaons. Detectors of Kaos consist of three planes of plastic scintillators to get time of flight (TOF) and amount of energy deposited information, two planes of threshold type aerogel Cherenkov detectors to discriminate between pions and kaons, and lead wall with a thickness of 10 14 $\mathrm{cm}$ to absorb positron showers from the beam target. Well established magnetic spectrometers Spek-A and Spek-C were placed at the backward of the target to get a better signal-to-background ratio, since backgrounds concentrate in forward angles although pions from hypernuclei decay uniformly. Detectors of pion spectrometers consist of two sets of drift chambers to reconstruct tracks, two planes of plastic scintillators to measure timing, and a threshold-type gas Cherenkov detector to discriminate between pions and electrons.

In the experiment, typical beam current was adopted by $20 \mu \mathrm{A}$. The total collected charge was $20 \mathrm{C}$ in 2 weeks. 


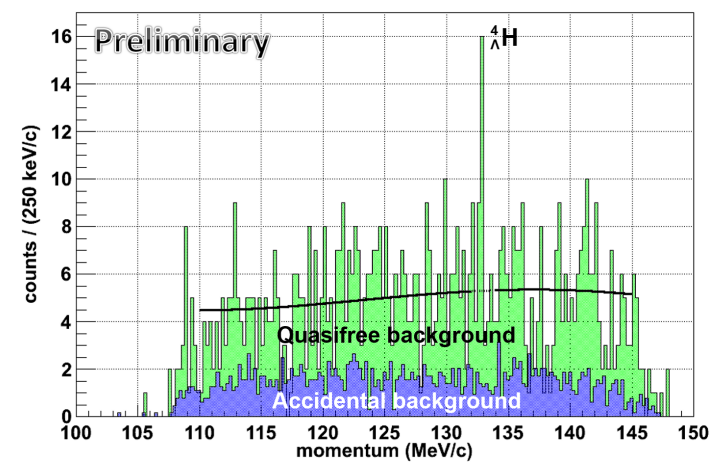

Figure 2: The momentum distribution of the decayed pion from hyperons and hypernuclei. The pions from ${ }_{\Lambda}^{4} \mathrm{H}$ were identified at $133 \mathrm{MeV} / \mathrm{c}$ in Spek-C, which is consistent with emulsion experiment [4]. The peak significance was obtained by the number of counts in two bins around $133 \mathrm{MeV} / \mathrm{c}$. The accidental background was obtained from off-coincidence events, and was scaled by the width of time window. The shape of the quasifree background was estimated by results of a Monte Carlo simulation including angular and energy dependences of kaons [15] and Fermi motion of nuclei in ${ }^{9} \mathrm{Be}$ target [14]. The normalization was obtained by a maximum likelihood fit ignoring the peak region and taking into account the accidental background.

\section{Results}

Figure 2 shows momentum distribution in Spek-C after selecting kaons in Kaos, namely the momentum distribution of the pion events from hyperon decay. We successfully identified the ${ }_{\Lambda}^{4} \mathrm{H}$ candidate with a peak significance of $\sim 3$ sigma (allowing all momenta within the momentum acceptance of the spectrometer) on top of the continuous accidental background and quasifree hyperon decay events. The yield of quasifree hyperons can be explained by a estimation from elementary cross section. The pilot experiment showed that this experimental method is promising. However, as quasifree background is not negligible, we have to get not only higher yield but also better background suppression.

\section{Future plan}

We are planning to perform an updated experiment with better suppression of quasifree background. A collimator which allows pion to pass from the target but blocks them from outside of the target will be installed to suppress the contamination of quasifree background. Figure 3 shows a result of Monte Carlo simulation for the momentum distribution of quasifree hyperons. It includes the angular and energy dependence of kaons and Fermi motion in the same way as Figure 2 About $70 \%$ reduction of quasifree background is expected around $133 \mathrm{MeV} / \mathrm{c}$ with the collimator. In addition, Spek-A will also cover the momentum around $133 \mathrm{MeV} / \mathrm{c}$ to get higher yield of ${ }_{\Lambda}^{4} \mathrm{H}$. We plan to perform next generation experiment in early summer of 2014.

\section{Summary}

We have performed two experiments of hypernuclear decay pion spectroscopy at MAMI-C in 2011 and 2012 to establish this new experimental technique. These experiments aim at the mass 


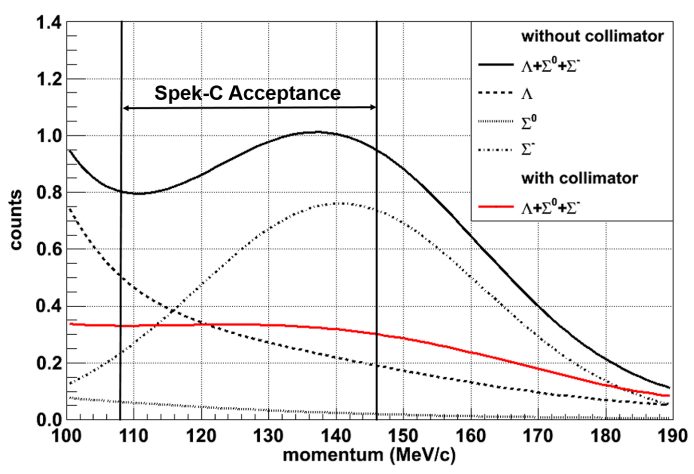

Figure 3: Expected momentum distribution of pions from hyperon decay. It is normalized by the counts of quasifree background at $133 \mathrm{MeV} / \mathrm{c}$ with the experiment in 2012. The momentum acceptance of Spek-C was also shown in the histogram. The background is expected to be reduced by $\sim 70 \%$, after the selection of $\pm 5 \mathrm{~mm}$ with respect to the direction of the electron beam at the target using collimator.

spectroscopy of hypernuclei with an accuracy of $30 \mathrm{keV} / \mathrm{c}^{2}$. A peak of ${ }_{\Lambda}^{4} \mathrm{H}$ events was identified and it proved that the experimental method is promising. We will perform an improved experiment in 2014 to obtain higher yield and less background.

\section{Acknowledgments}

This program is partially supported by the Strategic Young Researcher Overseas Visits Program for Accelerating Brain Circulation (R2201) and the core to core program of Japan Society for Promotion of Science (21002). This work was supported in part by the Federal State of RhinelandPalatinate, by the Deutsche Forschungsgemeinschaft with the Collaborative Research Center 1044, by the Carl Zeiss Foundation, and by the Research Infrastructure Integrating Activity 'Study of Strongly Interacting Matter' HadronPhysics3 (SPHERE) under the 7th Framework Program of EU.

\section{References}

[1] R. Machleidt et al., Phys. Rev. C 53 , R1483 (1996).

[2] R.B. Wiringa et al., Phys. Rev. C 51 , 38 (1995).

[3] V.G.J. Stoks et al., Phys. Rev. C 49 , 2950 (1994).

[4] M. Juric et al., Nucl. Phys. B 52 1-30 (1973).

[5] R.A.Brandenburg et al., Phys. Rev. C 37 781-785 (1988).

[6] A.Nogga et al., Phys. Rev. Lett. 88, 172501 (2002).

[7] E. Hiyama et al., Phys. Rev. C 65, 011301 (2002).

[8] A.R. Bodmer and Q.N. Usmani, Phys. Rev. C 31, 1400 (1985).

[9] S. N. Nakamura et al., Phys. Rev. Lett. 110012502 (2013).

[10] M. Bedjidian et al., Phys. Lett B 83 (1979) 252

[11] E. Hiyama et al., Phys. Rev. C 80054321 (2009).

[12] A. Margaryan, S.N. Nakamura, J. Reinhold, L. Tang, L. Yuan et al., JLab E08-012 Proposal (2008).

[13] K. I Blomqvist et al., Nucl. Inst. Meth. A 403 263-301 (1998).

[14] A. Bodek and J.L. Ritchie, Phys. Rev. D 23 1070-1091 (1981).

[15] T. Mart and C. Bennhold, Phys. Rev. C 61, 012201(R) (1999); T. Mart, ibid. 62, 038201 (2000); C. Bennhold, H. Haberzettl, and T. Mart, arXiv:nucl-th/9909022; T. Mart, C. Bennhold, H. Haberzettl, and L. Tiator, http://www.kph.uni-mainz.de/MAID/kaon/kaonmaid.html. 